\title{
Soft Set Theory and Its Application
}

\author{
Review By \\ DawaOngmu Sherpa \\ 20MSM2003 \\ ongmusherpa766@gmail.com \\ Department of M.Sc. Statistics
}

\begin{abstract}
Decision making is one of the everyday practices that we do in our day-to-day life and the process of selecting the best from the list of alternatives available for selection is called decision making. In this paper, we deal with the application of soft set theory (due to Molodstov 1999) due to decision making problems that is based on the rough reduction of parameters to keep the optimal choice objects.
\end{abstract}

Keywords:soft set, Reduct soft set, Choice value, Decision making

\section{Introduction}

Most of ourreal-life problems in the field of engineering, social and medical sciences, economics etc. involve imprecise data and their solution involves the use of mathematical principles based on uncertainty and imprecision. To overcome such uncertainties a number of theories have been proposed for dealing with such systems in an very effective way. Some of these are probability, fuzzy sets, intuitionistic fuzzy sets, interval mathematics and rough sets etc. All these theories, however, are associated with an inherent limitation, which is the inadequacy of the parameterization tool associated with these theories.And to overcome this problem the concept of soft set was first formulated by Molodtsov (1999) as a completely new mathematical tool for solving problems dealing with uncertainties.Molodtsov [1999] defines a soft set as a parameterized family of subsets of universe set where each element is considered as a set of approximate elements of the soft set. In the past few years, the fundamentals of soft set theory have been studied by various researchers. Maji et al. [2003] presented a detailed theoretical study of soft sets which includes subset and super set of a soft set, equality of soft sets, operations on soft sets such as union, intersection, and operations among others. He defines a soft set as a parameterized family of subsets of universe set where each element is considered as a set of approximate elements of the soft set. A soft set is a collection of approximate descriptions of an object. Each approximate description has two parts: a predicate and an approximate value set. In classical mathematics, we construct a mathematical model of an object and define the notion of the exact solution of this model. Usually, the mathematical model is too complicated and we cannot find the exact solution. So, in the second step, we introduce the notion of approximate solution and 
calculate that solution. In the Soft Set Theory, we have the opposite approach to this problem. The initial description of the object has an approximate nature, and we do not need to introduce the notion of exact solution. Due to the absence of any restrictions on the approximate description in Soft Set Theory makes this theory very easy and applicable in solving problems. The parameter is independent so we can use word or sentences as it will not create any problems in soft set theory because it is free from parameterization.

In this paper we use the concept of soft set, fussy set relation concepts and then apply in a decision-making problem for getting proper result. In approximate reasoning, fuzzy soft relations have shown to be of a primordial importance. Human judgements are often based on comparison between couples of faced data. Molodtsov gave definition of fuzzy soft relation. Afterwards Chaudhuri gave extended definition of fuzzy soft relation. Here we discuss the concept of soft relation and fuzzy soft relation and the definition of Chaudhuri in the simplified form as an algorithm and apply then in decision making problem taking another separate example.

\section{PRELIMINARIES}

In this section we present the notion of soft set which was introduced by Molodtsov 1999 to deal with uncertainty in non -parametric manner. A soft set is a collection of approximate description of an object.A soft set is a parameterised family of sets - intuitively, this is "soft" because the boundary of the set depends on the parameters. Formally, a soft set, over a universal set $X$ and set of parameters $E$ is a pair $(f, A)$ where $A$ is a subset of $E$, and $f$ is a function from $A$ to the power set of $X$. For each $e$ in $A$, the set $f(e)$ is called the value set of $e$ in $(f, A)$.

\section{Definition of soft set:}

Let $U$ be an initial universal set and $E$ a set of parameters or attributes with respect to $U$. Let $P(U)$ denote the power set of $U$ and $A \subseteq E$. A pair $(F, A)$ is called a soft set over $U$, where $F$ is a mapping given by $F: A \rightarrow P(U)$. In other words, a soft set $(F, A)$ over $U$ is a parameterized family of subset of $U$.

\section{Analysis}

The infectious disease COVID 19 has led to a dramatic loss of human life. It is a global pandemic which has not been over yet. It effects different people in different ways. Many of the preventative measure has been establish to protect our lives. Perhaps the most important fact that medical experts agree is that one of the best ways to stay healthy and protect ourselves is wearing a clean and proper face mask. As there are n number of face mask available in market the individual is unable to choose and make a correct decision on it, so here we apply the concept of fuzzy soft set in order to choose best among rest. 
The following types of face mask are used for decision making to select the best possible face mask as per need of the people.

h1, h2, h3, h4, h5, h6

The details of parameters are as follows:

- $\quad$ a. Expensive $=1$ and no $=0$

- b.Cottony soft $=1$ and no $=0$

- c. Washable, yes $=1$ and no $=0$

- d. Family safe, yes $=1$ and no $=0$

- e. Frequently available, yes $=1$ and no $=$

- f. Durable, yes $=1$ and no $=0$

Here we do a rough approach in order to choose the most efficient face mask as per the above requirement and the choice of the customer

Let $U=\{h 1, h 2, h 3, h 4, h 5, h 6\}$ be a set of six face mask, $E=$ \{expensive,cottony soft, washable, family safe, frequently available and durable $\}$ be a set of parameters.

Consider the soft set $(U, E)$ which describes the effectiveness of the face mask, given by (U, $E)=\{$ expensive $=\Phi$, cottony soft $=\{h 1, h 2, h 3, h 4, h 5, h 6\}$, washable $=\{h 1, h 2, h 6\}$, family safe $=\{h 1, h 2, h 3, h 4, h 5, h 6\}$, Frequently available $=\{h 1, h 2, h 3, h 4, h 6\}$, durable $\{h 1, h 3, h 6\}$.

Let say any family or organisation wanted to purchase a huge quantity on the basis of the parameter from the above example which constitute the subset $P=\{$ cottony soft, washable, durable, stretchable, family safe, good layers and Frequently available $\}$ of the set $E$. That means out of available items in $U$, buyer selects that item which qualifies with all or maximum number of parameters of the given soft set $P$.

\section{Tabular form of soft set}

The soft set $(F, P)$ on the basis of subset $P$ according to the choice of Mrs $X$ can be represented in the following table 


\begin{tabular}{|l|l|l|l|l|l|}
\hline U & e1 & e2 & e3 & e4 & e5 \\
\hline h1 & 1 & 1 & 1 & 1 & 1 \\
\hline h2 & 1 & 1 & 1 & 1 & 0 \\
\hline h3 & 1 & 0 & 1 & 1 & 1 \\
\hline h4 & 1 & 0 & 1 & 1 & 0 \\
\hline h5 & 1 & 0 & 1 & 0 & 0 \\
\hline h6 & 1 & 1 & 1 & 1 & 1 \\
\hline
\end{tabular}

\section{Reduction of soft set}

Consider a soft set $(F, E)$. Clearly for any $P C E,(F, P)$ is a soft subset of $(F, E)$. The tabular representation of the soft set $(F, P)$. When $R$ is a reduction of $P$, then the soft set $(F, R)$ is called the reduct soft set of the soft set $(F, P)$.

Choice value of $\mathrm{pi}$

The choice value of an object pi $€ U$, is $l_{i}$ given by

$$
l_{i}=\Sigma_{j} P_{i j}
$$

where $l_{i}$ are the entries in the table of the reductsoft-set

Algorithm for the selection of face mask which Mrs X wanted to buy

Input the set $\mathrm{P}$ of choice parameters of Mr. A which is a subset of $\mathrm{E}$.

- Input the soft set (F, E).

- Input the set $\mathrm{P}$ of choice parameters of Mrs $\mathrm{X}$ which is a subset of $\mathrm{E}$.

- Find all reduct soft sets of (F, P).

- Choose one reduct soft set say (F,R) of (F, P),

- $\quad$ Find $\mathrm{k}$, for which $l_{k}=\max l_{i}$

Then we can say that $l_{k}$ has optimal choice if $\mathrm{k}$ has more value than 1 then $\operatorname{Mrs} \mathrm{X}$ is independent of choosing any value from the options.

Clearly, from the table it is identified that $\{e 1, e 2, e 4, e 5\},\{e 2, e 3, e 4, e 5\}$ are the two reduces of $\mathrm{P}=\{\mathrm{e} 1, \mathrm{e} 2, \mathrm{e} 3, \mathrm{e} 4, \mathrm{e} 5\}$. Choose any one say, $\mathrm{R}=\{\mathrm{e} 1, \mathrm{e} 2, \mathrm{e} 4, \mathrm{e} 5\}$. From the choice value, we now get the reduce soft set as 


\begin{tabular}{|l|l|l|l|l|l|}
\hline U & e1 & e2 & e4 & e5 & $\begin{array}{l}\text { Choice } \\
\text { value }\end{array}$ \\
\hline h1 & 1 & 1 & 1 & 1 & L1=4 \\
\hline h2 & 1 & 1 & 1 & 0 & L2=3 \\
\hline h3 & 1 & 0 & 1 & 1 & L3=3 \\
\hline h2 & 1 & 0 & 1 & 0 & L4=2 \\
\hline h5 & 1 & 0 & 0 & 0 & L5=1 \\
\hline h6 & 1 & 1 & 1 & 1 & L6=4 \\
\hline
\end{tabular}

Here we get the maximum value $l_{i}=\mathrm{L} 1$ and $\mathrm{L} 6$ and from here the decision for purchasing best face mask by Mrs X could be either h1 or h6.

\section{Conclusion}

Since the soft set theory plays a very important role in every field of mathematics and also in real life related problems. Methods of this theory is very genuine and easy to understand for making decisions among different parameter. In this paper we applied the concept of fuzzy soft set for making decision among the face mask for preventing the risk of COVID-19.

\section{References}

[1] Molodtsov.D,Soft set theory-first result, Comput. Math. Appl, 37, pp.19-31,1999.

[2] Maji.P.K,Roy.A.R ,An Application of Soft Sets in A Decision Making Problem,Computers and Mathematics with Applications 44 ,pp.1077- 1083,2002.

[3] Xiao.Z.L, Chen. B, Zhong and Ye.S, Recognition for soft information based on the theory of soft sets, In: J. Chen, eds., Proceedings of ICSSSM-05, 2, pp. 1104-1106,2005. Soft set theory and its application-Research gate.

[4] Kharal.A and Ahmad.B, Mappings on Fuzzy Soft Classes, Hindawi Publishing Corporation, Advances in Fuzzy Systems Vol 2009, Article ID 407890, 6pages, 2009.

[5] Cagman.N and Enginogh.S, Soft set theory and uni-int Decision making, European journal of operational research 207,2,pp.848-855,2010.

[6]D. V. Kovkov, V. M. Kolbanov and D. A. Molodtsov, Soft sets theory-based optimization, J. Comput. Sys. Sc. Int., vol. 46(6), pp. 872-880, 2007. 
[7] P. Majumdar and S. K. Samanta,Similarity measure of soft sets, New. Math. Nat. Comput., vol. 4(1), pp. 1-12, 2008.

[8] R.K. Bhardwaj, S.K. Tiwari and Kailash Chandra Nayak, A Study of Solving Decision Making Problem using soft set, IJLTEMAS, Vol. IV, Issue IX, pp. 26-32, 2015.

[9] D. Chen, E. C. C. Tsang, D. S. Yeung and X. Wang, The parameterization reduction of soft sets and its applications, Compute. Math. Appl., 49 (2005), 757-763.

[10]https://www.researchgate.net/publication/326561107_Soft_Set_Theory_and_Its_A pplications

[11] http://www.elsevier.com/locate/camwa

[12] A-Study-Of-The-Fundamentals-Of-Soft-Set-Theory.pdf (ijstr.org) 